\title{
Autoprotection et protection émotionnelles: perspectives des enfants sur leur adaptation face au cancer et à la chimiothérapie de leur mère
}

\author{
Par Ann Hilton et Kris Gustavson
}

\section{Abrégé}

$P e u$ de recherches ont examiné les perceptions des enfants sur leur vécu lorsque leur mère était diagnostiquée d'un cancer du sein et suivait un traitement de chimiothérapie. Ce projet de recherche avait pour but de décrire les perspectives des enfants et de suggérer des interventions qui aideraient ces derniers à gérer l'expérience d'une manière moins stressante. On a fait appel à des méthodes d'enquête naturalistes qualitatives. On a utilisé un échantillonnage raisonné pour recruter des enfants dont la mère avait subi une chimiothérapie pour un cancer du sein au cours des deux années précédentes. On a ainsi interviewé onze enfants soit individuellement soit avec leurs frères et/ou sœurs. Les enfants avaient entre sept et 21 ans. Le principal thème qui est ressorti de l'étude est l'autoprotection et la protection émotionnelles. Par "autoprotection émotionnelle", on entend la manière dont les enfants se protégeaient de leurs pensées et sentiments et dont ils se protégeaient vis-à-vis d'autrui. Le terme "protection émotionnelle", lui, se rapporte à ce que les autres faisaient pour protéger émotivement les enfants. L'autoprotection et la protection émotionnelles renfermaient toutes deux des éléments concernant les domaines Conscience/Compréhension, Reconnaissance/Sentiments, Partage, et enfin, Changement/Aide. On propose des recommandations visant à aider les enfants, les parents, les infirmières et d'autres professionnels de la santé.

Le diagnostic et le traitement d'un cancer chez la mère et son hospitalisation représentent de graves menaces pour les enfants et se traduisent souvent par de l'anxiété et des conséquences négatives sur la vie familiale, la vie scolaire, les activités sportives et les loisirs (Hilton et Elfert, 1996; Nelson et al., 1994; Rosenfeld et Caplan, 1983; Zahlis, 2001). Bien que les gens soient de plus en plus sensibilisés à l'impact d'un cancer parental sur les enfants, certaines lacunes subsistent. L'incidence d'un cancer parental a souvent été examinée depuis la perspective du parent (Howe, Hoke, Winterbottom et Delafield, 1994; Hymovich, 1993; Lewis, Zahlis, Shands, Sinsheimer et Hammond, 1996; Lichtman et al., 1984; Shands, Lewis et Zahlis, 2000) et il convient de promouvoir les recherches se penchant sur la perspective de l'enfant. En outre, les études effectuées sur l'adaptation des enfants portaient sur des enfants et sur des adolescents plus âgés et ce, de deux à cinq ans après le traitement de leur mère (Brown, 1992; McTaggart, 2000; Nelson et al., 1994; Rosenfeld et Caplan, 1983). On n'a trouvé que deux études qui s'intéressaient à la période du diagnostic et du traitement (Hilton et Elfert; Zahlis). La présente étude visait à décrire les expériences des enfants lorsque leur mère devait suivre une chimiothérapie pour un cancer du sein. Une sensibilisation accrue aux perspectives des enfants permet de mieux comprendre leur vécu et d'orienter l'élaboration de méthodes permettant de faciliter l'adaptation des enfants à la maladie de leur mère.

\section{Écrits pertinents}

$\mathrm{Si}$ on veut comprendre le vécu des enfants, il importe que leur participation à des études soit pour eux une occasion valable d'être entendus. Ainsi, il se peut que les entrevues nous aident à mieux comprendre le phénomène que les questionnaires structurés. Les études auprès d'enfants aux prises avec un cancer parental faisaient principalement appel à des entrevues auprès d'enfants (Brown, 1992;
Issel, Ersek et Lewis, 1990; Nelson, Sloper, Charlton et While, 1994; Rosenfeld et Caplan, 1983; Zahlis, 2001) et à des entrevues auprès de parents et d'enfants (Hilton et Elfert, 1996; McTaggart, 2000). Plusieurs autres comptaient sur des entrevues et des questionnaires structurés (Birenbaum, Yancey, Phillips, Chand et Huster, 1999; Compas et al. 1994; Wellisch, Gritz, Schain, Want et Siau, 1991 et 1992).

Lorsqu'un de leurs parents est atteint d'un cancer, les enfants s'inquiètent de plusieurs choses, notamment de ce qui va se passer, si leur mère va mourir, si les choses vont changer; ils se préoccupent au sujet de leur famille, s'ils devraient en parler à d'autres, de leur propre risque d'être atteints du cancer et de l'évolution de la situation financière de leur famille (Zahlis, 2001). Les chercheurs ont également appris des détails concernant le niveau de conscience et de compréhension des enfants, leurs besoins d'information et s'ils parlent ou non de la situation entourant le cancer. La conscience était un thème majeur de l'étude de Hilton et Elfert (1996). Il est évident que le niveau de développement de l'enfant exerçait une influence sur la compréhension et sur la nature des demandes faites. Les parents jugeaient que les enfants d'âge préscolaire étaient trop jeunes pour comprendre et ils leur donnaient des explications simples. Ils échangeaient davantage d'information avec les enfants d'âge scolaire qui croyaient que la situation était grave. Rosenfeld et Caplan (1983) ont constaté qu'il existait des différences considérables sur le plan de ce qu'on disait aux enfants et de ce qu'ils comprenaient et que les problèmes touchant les rapports parent-enfant étaient liés à la mesure dans laquelle les enfants étaient tenus au courant de l'évolution de la maladie. La plupart des enfants ne voulaient pas ou ne pouvaient pas en parler à qui que ce soit à cause d'une règle familiale interdisant toute discussion au sujet de la maladie et parce qu'ils estimaient que personne ne pourrait comprendre. Les enfants qui n'avaient pas reçu suffisamment d'information sont devenus anxieux et rancuniers et sont restés en colère. Nelson et ses collègues (1994) ont découvert que l'impossibilité dans laquelle se trouvaient les enfants de discuter avec les parents de la maladie du parent malade était reliée à une grande anxiété.

Des études présentent l'impact du cancer sur les routines et les habitudes de vie des enfants. La dépendance, un des thèmes majeurs définis par Hilton et Elfert (1996), reflétait les variations en matière de dépendance et d'indépendance selon les groupes d'âge. Les familles ayant des enfants d'âge préscolaire centraient leur attention sur les soins à l'enfant et sur l'emploi du temps. Les enfants d'âge scolaire manifestaient de la sollicitude envers leur mère et étaient plus autonomes que les tout-petits, mais ils dépendaient tout de même de leurs parents. Ils participaient aux tâches ménagères. Les adolescents, eux, ressentaient la menace et décrivaient une plus grande intimité émotionnelle. Ils se percevaient comme étant extrêmement occupés, ayant trop d'engagements à l'école et étaient irrités par toute demande additionnelle qu'on leur imposait à la maison. La maladie estompait les différences entre les rôles et certains des adolescents dispensaient

B. Ann Hilton, RN, PhD, est professeure à l'École des Sciences infirmières, Université de la Colombie-Britannique, Vancouver, C.-B.

Kris Gustavson, RN, MSN, est directrice des Services aux patients du Children's Centre, Mount Saint Joseph Hospital, Vancouver, $C .-B$. 
des soins pratiques et se chargeaient de tâches ménagères. Rosenfeld et Caplan (1983) ont également décrit l'ajout de tâches domestiques et de responsabilités relatives au soin des frères et sœurs et ont constaté que de nombreux adolescents étaient obligés d'abandonner certains sports et passe-temps. Ils passaient également moins de temps avec leurs amis ce qui représentait pour eux une source d'anxiété. Nelson et al. (1994) sont parvenus à la même conclusion et indiquaient que le haut degré d'anxiété était lié au fait que les adolescents devaient passer moins de temps avec leurs amis et consacrer moins de temps aux activités sportives et de loisir, à la détérioration de leur rendement scolaire et à l'anxiété continuellement ressentie à propos de la maladie.

Issel et ses collègues (1990) ont dégagé quatre thèmes reliés à la manière dont les enfants abordaient le cancer du sein de leur mère. Le thème "Dans ses souliers à elle" reflétait ce que les enfants voudraient s'ils étaient à la place de leur mère et des moyens pour eux d'anticiper ce dont elle pouvait avoir besoin. Les jeunes enfants étaient plus prévenants, prenaient bien soin de leur mère, participaient aux tâches ménagères et essayaient de bien se comporter. Le thème "La routine l'emporte" décrivait la manière dont les enfants minimisaient la gravité de la maladie en adoptant un comportement normal et en maintenant les habitudes. Quant au thème "Énergie collective", il montrait comment les enfants cherchaient un réconfort auprès des autres. Le dernier thème, "On discute de tout", décrivait ce que les enfants faisaient pour essayer de comprendre l'expérience de la maladie en résolvant des problèmes et en interprétant l'information. Entre autres choses, ils exprimaient tout haut leurs pensées et leurs sentiments par le bais de discussions en famille et de réflexions personnelles. Les enfants plus âgés étaient plus susceptibles de parler de la situation et d'y réfléchir.

McTaggart (2000) a exploré l'expérience du cancer du sein et la relation mère-fille adolescente. "Changement de paysage" décrivait la trajectoire sur le plan de l'expérience vécue et de la signification depuis le diagnostic jusqu'aux effets du traitement et se poursuivait dans le présent et l'avenir tel qu'il était imaginé. La mère et la fille gardaient secrètes leurs inquiétudes à propos du cancer et sur la possibilité que ce diagnostic frapperait également la fille un jour. "Avoir l'intention et faire semblant" décrivait la sollicitude et l'attitude protectrice mutuelles visant à atténuer la menace. Maintenir un sentiment de normalité et limiter les conversations relatives à l'expérience du cancer étaient deux stratégies courantes. "Acquisition de la sagesse" décrivait le changement personnel engendré par l'expérience. "Relations mère-fille durables" reflétait la qualité des relations mère-fille et l'importance du cancer dans leur relation.

L'étude de Brown (1992) s'intéressait aux adolescents vivant avec un parent atteint d'un cancer avancé. Cette situation avait un impact émotionnel et physique profond et les adolescents décrivaient "l'autoprotection émotionnelle" comme étant une stratégie qui leur permettait de se tourner vers les tâches liées à l'adolescence et de poursuivre leur cheminement, en dépit de la détérioration de la situation à la maison. Ils se protégeaient d'une réalité pour laquelle ils n'étaient pas prêts. Un thème sous-jacent se rapportait au désir des adolescents de mettre leur vie avant celle des autres et de se concentrer sur le travail qui accompagne cette période de la vie. Ils se protégeaient émotivement en choisissant de ne pas penser à la situation, en gardant une attitude positive et en essayant de voir leur père ou leur mère comme il ou elle était avant que la maladie ne survienne. Les plus jeunes des adolescents étaient les plus résolus à ne pas penser à la possibilité du décès de leur parent et ils se sentaient davantage prisonniers et rancuniers que les plus âgés des adolescents qui estimaient, eux, qu'ils avaient davantage de responsabilités.

Les thèmes communs se dégageant de ces études montrent que le cancer est une menace majeure qui a des répercussions sur de nombreuses dimensions de la vie des enfants et que la conscience et la compréhension qu'ils ont de la maladie varient grandement de même que le fait de parler de leurs inquiétudes. On peut minimiser la gravité de la maladie et limiter les perturbations qu'elle entraîne afin que la vie des enfants demeure aussi normale que possible. Ces études améliorent la compréhension que l'on a du vécu des enfants, mais peu de recherches ont été effectuées peu de temps après le traitement, particulièrement lorsque la chimiothérapie est le principal traitement utilisé. L'étude tentait de répondre à la question suivante: "Quelle est l'expérience des enfants lorsque leur mère doit subir une chimiothérapie pour son cancer du sein?" Cette étude faisait partie d'une étude plus vaste dans le cadre de laquelle l'équipe de recherche travaillait en coopération avec les femmes atteintes, leur partenaire masculin et leurs enfants pour comprendre ce qui favorisait ou au contraire entravait l'adaptation et pour identifier des moyens de les aider à vivre cette expérience de manière moins stressante (Hilton et al., 1996).

\section{Méthodes}

L'équipe de recherche se composait d'infirmières, de travailleurs sociaux, d'un oncologue et de femmes atteintes du cancer du sein et d'un conjoint. Nous avons retenu, pour la plus vaste étude, des méthodes de recherche-action qualitatives de nature participative du fait de l'accent mis sur la collaboration et l'action et sur l'élaboration de stratégies (Erlandson, Harris, Skipper et Allen, 1993). Au sein de l'équipe de recherche, les femmes atteintes de cancer du sein et le conjoint ont participé à l'élaboration du protocole de recherche et à la discussion des résultats. Dans la partie consacrée aux enfants, l'accent était uniquement mis sur la participation de l'enfant au processus d'entrevue. Des entrevues semi-dirigées ont été réalisées auprès de 11 enfants et ce, à leur domicile. En général, les frères et sœurs préféraient être interviewés tous ensemble. Étant donné que le but primordial de l'étude n'était pas d'établir des distinctions entre les réponses des enfants interviewés individuellement ou en groupe au niveau des opinions, on a respecté leur préférence en la matière. Cette décision peut avoir influé sur la communication de leurs perspectives. Les entrevues semi-dirigées exploraient les perceptions de l'expérience vécue des enfants et ce qui favorisait ou au contraire entravait leur adaptation face à la maladie. Par exemple, on demandait aux enfants ce qui leur est venu en tête quand on leur a annoncé, la toute première fois, ce qui arrivait à leur mère, ce qu'on leur avait dit et ce qu'ils ressentaient quand leur mère subissait son traitement de chimiothérapie. On cherchait avant tout à comprendre le vécu des enfants lorsque leur mère devait subir une chimiothérapie, et non à séparer l'impact particulier de la chimiothérapie sur leur adaptation. L'étude a été approuvée par les comités d'éthique concernés.

On a fait appel à un échantillonnage raisonné type boule de neige, d'enfants dont la mère avait au moins un enfant à la maison et avait suivi une chimiothérapie pour son cancer du sein au cours des 24 mois précédant la tenue de l'étude. Le personnel du centre de cancer a tout d'abord communiqué avec les familles qui répondaient aux critères de l'étude. Si les familles y consentaient, elles étaient contactées par l'équipe de recherche. D'autres participants ont été recrutés dans le cadre des contacts personnels de membres de l'équipe de recherche. Onze enfants appartenant à six familles y ont participé. Les familles comptaient de un à quatre enfants $(\mathrm{M}=2,3)$, dont l'âge allait de quatre à 28 ans $(\mathbf{M}=12,3$ ans $)$. Les enfants interviewés avaient de sept à 21 ans et il y avait parmi eux quatre garçons et sept filles. Quatre enfants étaient de jeunes écoliers (sept à neuf ans), deux étaient des écoliers plus âgés (10-12 ans), trois étaient de jeunes adolescents et le dernier, un adolescent plus âgé (17-19). L'échantillon incluait également un jeune homme de 21 ans bien qu'il n'appartienne pas à un des groupes d'âge retenus parce que l'occasion s'est présentée et parce que nous avons pensé que sa contribution serait précieuse. Sa mère avait subi plusieurs cycles de chimiothérapie en l'espace de quelques années et elle était aux prises avec une grave récidive de son cancer.

La plupart des mères étaient ou avaient été enseignantes, professionnelles de la santé, techniciennes, professionnelles ou femmes au foyer. Six pères détenaient une formation universitaire. La plupart des familles avaient deux revenus et le revenu familial annuel se situait entre 36000 et 95000 \$ et plus. De six à 25 mois s'étaient 
écoulés entre la chimiothérapie de la mère [moins de six mois (deux), six - 12 mois (deux), 16 - 25 mois (sept)]. Une femme était entrée dans la phase palliative de la maladie.

Les entrevues ont été enregistrées sur cassette puis retranscrites, et les transcriptions ont fait l'objet d'une lecture attentive. On a utilisé un codage ouvert afin de dégager les idées, et une analyse additionnelle a permis de cerner les thèmes d'ordre supérieur. On s'est servi d'une analyse comparative continue à des fins de clarification et de raffinement additionnels (Strauss et Corbin, 1990). On en a établi la fiabilité et la rigueur en clarifiant la piste décisionnelle (vérifiabilité), en restant fidèles aux propos des répondants, en utilisant des citations pour présenter les résultats (crédibilité) et en discutant avec l'équipe et d'autres personnes des thèmes tels qu'ils ont été dégagés ainsi que des détails à l'appui (convenance) (Denzin et Lincoln, 1994; Lincoln et Guba, 1985). Ce processus a favorisé la détermination des propriétés et des dimensions et a permis de vérifier la validité de l'ajustement.

\section{Résultats}

L'étude a fait ressortir deux thèmes majeurs à savoir l'autoprotection émotionnelle et la protection émotionnelle. L'autoprotection émotionnelle se rapporte à l'ouverture ou à la protection dont les enfants faisaient preuve sur le plan de leurs pensées et sentiments et de la manière dont ils se protégeaient émotivement ou au contraire s'ouvraient aux autres. Par protection émotionnelle, on entend ce que les personnes autour d'eux faisaient pour protéger les enfants de la situation et du stress qui l'accompagnait ou au contraire pour qu'ils s'ouvrent à leur sujet. L'étude a également permis de cerner les sous-thèmes "Conscience/Compréhension," "Reconnaissance/Sentiments," "Partage" et "Changement/Aide". Le tableau un présente ces thèmes et la dimension d'ouverture/de protection au sein de chacun.

\section{Conscience/Compréhension}

"Conscience/Compréhension" reflète le degré de conscience et de compréhension qu'ont les enfants de la situation ou leur absence, leurs attentes relatives à l'avenir et ce qu'ils faisaient pour essayer de développer leur compréhension ou pour se protéger de l'information. La compréhension était sous la haute influence de ce que les autres leur disaient, à condition qu'on leur dise quelque chose. Certains enfants se protégeaient émotivement de l'information alors que d'autres la recherchaient activement. Certains étaient protégés de l'information tandis que d'autres étaient tenus informés de la situation.

Les enfants manifestaient divers niveaux de conscience et de compréhension de la situation de leur mère. En général, le degré de conscience était moindre chez les plus jeunes. "J'étais seulement en première année et je ne savais pas vraiment ce qui allait se passer." Un autre garçon a souligné le faible degré de conscience qu'il avait: "Je n'avais pas une idée précise de la gravité de la situation... alors, je ne pense pas que cela me tracassait." Même un adolescent plus âgé indiquait ne pas savoir ce qui se passait au début. En revanche, plusieurs enfants, dont certains étaient assez jeunes, manifestaient une compréhension modérée à complète de la situation et de ses conséquences potentiellement graves. Ainsi, deux sœurs âgées de sept et neuf ans ont dit: "Le cancer est une sorte de cellule qui contamine le corps, qui infecte les bonnes cellules... et un peu de cancer a pénétré dans ses côtes" et "je sais qu'elle pourrait en mourir." Leur mère leur avait expliqué la situation avec une grande délicatesse. "Si maman meurt, elle m'a dit que Dieu lui donnerait probablement une étoile spéciale pour qu'elle s'y assoie près de la fenêtre de notre chambre." Les adolescents avaient généralement conscience que la situation était préoccupante et qu'il y avait une chance que leur mère meure: "Le cancer tue et elle pourrait mourir et on se retrouverait alors seuls." Une autre adolescente a développé une meilleure compréhension de la situation après avoir suivi un cours de biologie mais “qu'au début, lorsqu'elle me parlait des ganglions lymphatiques
- je ne savais pas de quoi il s'agissait. Tout ce que je savais, c'est que c'était une mauvaise nouvelle."

Il était difficile de se faire une image claire de la compréhension qu'avait le jeune homme de 21 ans au sujet des récidives du cancer de sa mère. Elle avait été très malade peu avant et "souffrait d'une importante accumulation de liquide." Il se demandait quel serait son état de santé à l'avenir, mais il ne souhaitait pas en dire plus quoiqu'il croie qu'elle irait "bien". Au cours de l'entrevue familiale qui a suivi, sa mère insistait que seules les pensées positives étaient acceptables, ce qui influençait sûrement son fils à ne pas révéler ses préoccupations majeures.

Les enfants restaient optimistes et espéraient que les choses allaient bien se passer mais cela ne les empêchait pas de s'inquiéter et de craindre que leur mère ne meure. Ils avaient de la difficulté à parler de la gravité de sa maladie et sentaient qu'ils ne pouvaient rien faire pour changer la situation: "Il n'y a rien que nous puissions faire pour améliorer les choses." Un garçon plus pessimiste que les autres répondants a expliqué que l'idée de la mort éventuelle de sa mère ne l'avait pas préoccupé lorsque qu'elle suivait sa chimiothérapie, mais qu'il traversait une phase plus négative et qu'il croyait dorénavant que sa mère allait mourir. Il a ajouté qu'il savait plus de choses qu'avant. "Le cancer est partout... Je m'inquiète lorsqu'elle est fatiguée, lorsqu'elle fait des choses, lorsqu'elle soulève des objets qu'elle n'est pas censée soulever." Ses inquiétudes et ses peurs augmentaient car il connaissait d'autres personnes qui avaient eu le cancer et en étaient mortes.

La compréhension et les réponses des enfants étaient grandement influencées par ce que d'autres personnes leur avaient dit et la manière utilisée pour le dire et par ce qu'ils lisaient et entendaient. Certains parents leur expliquaient la situation ouvertement dans des termes simples tandis que d'autres protégeaient émotivement leurs enfants en limitant l'information qui leur était communiquée. En général, les enfants ont trouvé pénible l'annonce de la maladie de leur mère, mais si on leur disait que sa lutte pour retrouver la santé se passait bien et que "elle ne va pas mourir", ils éprouvaient du soulagement et souvent, ne désiraient pas en savoir davantage, se protégeant ainsi de toute information additionnelle. Un jeune adolescent a déclaré plutôt passivement: “je comprenais les choses qu'on me disait... qu'elle s'en remettrait et qu'elle reviendrait bientôt à la maison (et il ne voulait pas qu'on lui donne davantage d'information)."

Les parents ont fait appel à diverses méthodes pour informer leurs enfants mais ce sont les images et les films vidéos sur le cancer et l'adaptation au cancer qui s'avéraient les plus efficaces. Une fille de 10 ans a signalé que le fait de regarder des vidéos en famille l'avait aidée. D'autres familles ont encouragé leurs enfants à prêter attention à ce qui se passait. Quelques enfants sont allés à la clinique de cancérologie et ils avaient généralement trouvé cela utile. D'autres enfants s'étaient vus priés par leur père "d'être silencieux" lorsqu'ils se rendaient à l'hôpital et ils "restaient assis dans la chambre sans rien faire d'autre". En l'absence de conseils ciblés, l'expérience n'était ni très positive ni très utile. Des adolescentes plus âgées se souvenaient bien de la tension qui caractérisait leurs visites à la clinique d'oncologie:

Notre père ne voulait pas qu'on y aille. Mais moi, je voulais y

aller. Je parlais à maman et je jouais avec ses cheveux. Il s'est

fâché après moi. Il m'a dit, 'Ne lui touche pas.' Il ne sait pas quoi faire... J'ignore si c'est parce qu'il ne voulait pas qu'on la voie dans cet état et qu'il n'osait pas nous le dire.

Souvent, les enfants qui avaient le plus fortement conscience de la situation se consacraient entièrement au maintien des habitudes familiales et se trouvaient ainsi constamment confrontés à la réalité. Il n'était pas inhabituel que les enfants se protègent émotivement en contrôlant ce qu'ils savaient. Tandis que certains limitaient l'entrée d'information en évitant les situations où le sujet pourrait être abordé et les gens qui pourraient en parler, d'autres se mettaient activement en quête d'information. Cependant, la pertinence, l'exactitude et l'utilité de l'information n'étaient pas toujours évidentes et elles peuvent avoir engendré de la confusion. Une adolescente plus âgée a obtenu des 
Tableau un: Schémas de l'autoprotection émotionnelle* et de la protection émotionnelle**

\section{Conscience/Compréhension}

Le degré de conscience et de compréhension qu'ont les enfants de la situation ou leur absence, leurs attentes relatives à l'avenir et ce qu'ils faisaient pour essayer de développer leur compréhension ou pour se protéger de l'information. Ce que d'autres personnes faisaient pour les informer ou ne pas les informer.

\section{Autoprotection émotionnelle}

1. Naïveté/faible conscience, compréhension limitée

2. La compréhension/le souci sont à un niveau raisonnable, ouverture à l'information

3. Trop d'information/de confusion/manque de réalisme; cherche à obtenir trop d'information, ne sait pas quoi en faire

Protection émotionnelle

1. N'est pas mis au courant de la situation

2. Bien informé pour son niveau de développement

3. Reçoit trop d'information

\section{Reconnaissance/Sentiments}

La reconnaissance, par les enfants, de la tension présente chez eux et chez autrui (ou de son absence) et leur utilisation (ou non) de stratégies de soutien.

\section{Autoprotection émotionnelle}

1. Faible conscience, focalisation sur soi, faire bonne figure, faire comme si tout va bien

2. Reconnaître les sentiments présents chez soi et chez autrui, reconnaissance/expression/partage des soucis, recherche et utilisation de mesures de réconfort

3. Focalisation sur les peurs, faible connaissance de soi, utilisation de stratégies inefficaces, passage à l'acte versus apaisement

\section{Protection émotionnelle}

1. Protégé du stress et des tensions familiales

2. Subit une exposition raisonnable au stress et aux inquiétudes

3. Aucune protection des tensions

\section{Partage}

La nature des conversations des enfants avec autrui sur la situation de leur mère et sur leurs inquiétudes ainsi que la mesure dans laquelle ils les tiennent.

\section{Autoprotection émotionnelle}

1. Repli sur soi-même, focalisation sur la tristesse

2. Partage raisonnable, liens profonds, humour

3. Conversations sans fin, pas de limites précises, humour non approprié

Protection émotionnelle

1. S'y attaque seul

2. La voie est frayée

3. Pas de pensées secrètes

\section{Changement/Aide}

La mesure dans laquelle les changements avaient un impact sur les enfants et la manière dont ces derniers aidaient à la maison.

\section{Autoprotection émotionnelle}

1. Rigidité dans le maintien des routines de vie habituelles, mise de côté du moi, ne pas savoir quoi faire/quoi dire

2. Routines de vie raisonnablement normales, acceptation des changements, établissement d'un bon équilibre famille-amis, bénéficie de conseils

3. Bouleversement des routines de vie, ressentiment envers le changement, sentiment de culpabilité, d'être dirigé et contrôlé par les autres

\section{Protection émotionnelle}

1. La vie continue normalement, ne s'implique pas dans la situation, contrôlé par les autres

2. S'adapte, coopère, a le sentiment d'aider, d'être reconnu

3. Effectue des changements majeurs, abandonne des facettes de sa vie, grandit à toute vitesse, ressent le poids d'une charge accablante, ressent de la rancœur, passe à l'acte

\section{Signification des niveaux 1, 2, 3:}

Niveau 1 - important degré de protection émotive chez les enfants ou venant des autres

Niveau 2 - degré modéré de protection émotive chez les enfants ou venant des autres

Niveau 3 - aucune protection émotive chez les enfants ou venant des autres

*Autoprotection émotionnelle - comment les enfants se sont protégés de leurs propres pensées et sentiments et de ceux d'autrui (et se sont ouverts aux autres)

**Protection émotionnelle - ce que d'autres personnes ont fait pour protéger les enfants de la situation (et les y exposer) 
renseignements à l'école mais, dans l'ensemble, elle avait l'impression de "savoir rien de rien". Plusieurs enfants ne désiraient pas en savoir davantage sur l'état de santé de leur mère quand elle a reçu son diagnostic et a suivi sa chimiothérapie. Ils estimaient que le fait d'en savoir davantage ne ferait qu'empirer les choses: "Je pense que si j'en avais su plus, les choses auraient été pires". Un garçon a commenté: "Je ne faisais pas attention à ce qui se passait, alors je ne sais pas vraiment."

Souvent, les enfants ne voulaient pas en parler et ils résistaient même activement à toute tentative de communication. "J'essayais de ne pas en parler. Papa en a parlé quelques fois. Il commençait en disant 'Si ta mère mourait,' et je répliquais tout de suite 'Ne me parle pas de ça!' Papa en parlait pour se préparer à faire face à la situation si cette éventualité se concrétisait; c'est pour ça qu'il en parlait." Sa jeune sœur a déclaré:

On ne peut pas s'empêcher d'y penser mais on ne le dit jamais...

Je ne pouvais pas prononcer ce mot-là. Je ne me souviens pas avoir dit ce mot... Je me mettrais probablement à pleurer. Je suppose que j'avais extrêmement peur. Cela ne va pas arriver. Je ne peux pas vivre sans elle. J'éprouvais une immense dépendance vis-à-vis de ma mère. J'ai toujours été proche d'elle et à la pensée qu'elle ne serait plus là... il ne me resterait plus

qu'à mourir moi aussi. Je ne peux pas vivre sans elle.

La sœur aînée de cette famille a alors déclaré: "ma mère m'avait dit ce que ma sœur pensait parce je suis beaucoup plus autonome. Mes parents croyaient que j'avais ce qu'il fallait pour faire face à la situation."

En revanche, certains enfants se mettaient en quête d'information. Les petites filles d'une mère qui recevait des soins palliatifs ont été informées de la situation et elles se sont tournées vers leurs parents pour leur poser des questions. "Il nous dit à peu près ce qui se passe même s'il y a des fois où il ne le fait pas, mais si on lui pose une question précise, il explique les choses parce que nos parents nous disent tout." Une fille a demandé à son père si sa maman allait mourir et il lui a répondu que c'était une possibilité. Ces filles jugeaient qu'elles faisaient bien de poser des questions. Dans une autre famille, le frère et la soeur demandaient conseil et posaient des "questions, etc." à leur soeur aînée qu'ils admiraient, en qui ils voyaient une personne plutôt sophistiquée et qui s'exprime avec aisance. Cette dernière ne se souvenait pas du fait qu'ils étaient venus la voir pour lui demander information ou conseils. Les enfants d'une autre famille voulaient "vérifier si le médecin n'était pas un raté de première classe." Deux adolescentes appartenant à une autre famille auraient voulu que la famille tienne une réunion avec le médecin afin qu'elles puissent apprendre ce qui "allait se passer", qu'elles se sentent plus à l'aise avec lui et qu'elles aient "la possibilité de s'adresser à lui si elles avaient des problèmes ou avaient besoin d'aide pour bien comprendre." Elles estimaient que le fait de parler avec un médecin serait très différent du fait de parler à un thérapeute, "ce n'est pas comme un thérapeute avec qui on partage ses sentiments et d'autres détails - ici, il s'agit simplement des faits pour savoir ce qui va se passer."

Quelques-uns des enfants recherchaient activement de l'information écrite. Une adolescente a cherché la définition du terme "cancer" dans un dictionnaire, une autre a fait de même dans une encyclopédie et un jeune adulte a obtenu de l'information à la bibliothèque. Il voulait savoir ce qu'il pourrait faire si quelque chose devait arriver à sa mère (mais il ne voulait pas donner plus de détails sur ce que cela signifiait). Seule une adolescente plus âgée s'est penchée sur le risque qu'elle courait d'être atteinte du cancer. "J'ai lu des livres sur ce sujet. Je voulais savoir quelle était ma situation à moi et j'ai regardé un documentaire. Il était déprimant, alors j'ai arrêté les recherches."

Pour essayer de comprendre la situation ou la replacer dans son contexte, certains enfants comparaient leur situation à celles d'autres enfants. Une adolescente jugeait que sa mère était plus malade que d'autres patientes, “ma maman, par rapport à d'autres femmes, elle n'allait vraiment pas bien. C'était un événement, un grand évènement lorsque maman nous rejoignait à table pour manger parce que cela voulait dire que sa journée se passait bien.” En général, les enfants ne disaient pas qu'ils se demandaient pourquoi leur mère avait le cancer, mais une adolescente voulait savoir pourquoi la maladie avait frappé sa mère. La jeune fille était "furieuse, rebelle et effrayée - répétant constamment "pourquoi”. Pourquoi pas quelqu'un d'autre qui était bien plus méchant?"

Ainsi, les enfants se protégeaient eux-mêmes et étaient protégés, émotivement, par leur entourage. Ils étaient réceptifs ou non, recherchaient de l'information ou préféraient ne rien savoir. Les parents et d'autres personnes exerçaient une grande influence, notamment auprès des jeunes enfants, sur le plan de ce qu'ils savaient et comment ils réagissaient à l'annonce du cancer.

\section{Reconnaissance/Sentiments}

"Reconnaissance/Sentiments" fait référence à la reconnaissance, par les enfants, de la tension présente chez eux et chez autrui et à leur utilisation de stratégies de réconfort ou de soutien. Ils ont parlé de leurs inquiétudes et de leurs peurs et de la manière dont ils composaient avec le stress. Leur degré de conscience et de compréhension influait sur leur réaction émotionnelle. Un grand nombre d'entre eux étaient protégés du stress par ce qu'on leur avait révélé ou pas et par les émotions qu'ils observaient chez autrui.

Certains enfants avaient une grande conscience de leur anxiété, de leurs peurs et inquiétudes alors que chez d'autres, elle semblait bien moindre. "En ce moment, je m'inquiète un peu de ce qui pourrait arriver, mais pas beaucoup." Deux soeurs adolescentes se tracassaient lorsque leur mère se rendait à un examen de santé: "Je ne veux pas la voir revenir à la maison comme ça - quand elle m'apprend que la maladie est revenue." Une petite fille avait de la difficulté à faire face à la douleur de sa mère. Les enfants étaient nombreux à être très conscients de leurs sentiments. Une petite fille dont la mère était en phase palliative a déclaré: "Quand maman a attrappé la maladie, j'ai pleuré, mais je ne pleure pas quand j’y pense à l'école." Tout comme sa petite soeur, elle était en colère contre la maladie "le cancer me rend furieuse."

Certains enfants étaient troublés par la perte de cheveux de leur mère et par les questions des gens à ce sujet. 'C'est pénible d'avoir devant soi une personne qui a eu tous ses cheveux pendant si longtemps et qui les perd tous, d'un seul coup." Une petite fille disait aux gens que la perruque servait à cacher la réalité. Un petit garçon trouvait cela "très bizarre parce qu'habituellement on ne voit que des hommes chauves." Un adolescent a dit que ses parents "ont réagi à la perte des cheveux bien plus sérieusement que moi." Un jeune adulte aidait sa mère à attacher ses foulards, une compétence qu'il avait acquise auprès de ses pairs. Il aidait aussi sa mère à acheter ses foulards et à les utiliser de manière novatrice afin que l'alopécie ne soit plus si évidente.

Plusieurs enfants percevaient l'augmentation de la tension au sein de la famille. "Je crois qu'on s'énervait davantage les uns les autres... on cherchait la petite bête... On ne se sentait pas bien alors on s'agaçait les uns les autres." Les enfants percevaient le stress et les exigences accrus qui accablaient leur père et la difficulté qu'il avait d'accomplir des tâches peu familières: "Je crois que pour papa le plus dur était de préparer le souper... de le mettre dans le four et de l'y laisser à la température qui convenait et pour le temps qu'il fallait." Les enfants réalisaient que c'était plus difficile pour les papas parce qu'ils n'avaient pas beaucoup fait la cuisine avant: "Papa ne sait pas du tout cuisiner." Alors que certains pères restaient plus souvent au foyer en vue d'aider, d'autres continuaient de se rendre au travail, tout en réduisant parfois leurs heures de travail. Quelques enfants ont perçu la tension qui rongeait leur père, l'importance que revêtait pour lui son emploi et l'occasion qu'il lui donnait de s'éloigner de la situation familiale. "Il éprouvait de la frustration et de la confusion et il souffrait à l'intérieur mais ce n'est pas le genre de personne qui montre ses émotions. Son lieu de travail est un endroit où il peut se relâcher... C'est un endroit sûr où il n'a pas besoin de s'inquiéter de toutes ces choses-là." Certains enfants avaient une vive conscience de 
la tension accrue qui régnait dans leur famille tandis que d'autres ne la percevaient pas, peut-être parce qu'ils étaient protégés émotivement par ce qu'on leur disait et par ce qu'ils voyaient autour d'eux. Il est possible que beaucoup de familles aient tenté d'épargner ce stress aux enfants en taisant leurs propres peurs.

Certains enfants essayaient eux aussi de ne pas communiquer leurs peurs et leurs inquiétudes en faisant bonne impression. Ils ne voulaient pas y penser; ils voulaient éviter le sujet et "faire comme si tout allait bien.” Deux soeurs n'en ont pas parlé, "faire semblant pour tout le monde, alors que tu n'es pas là.” Une adolescente plus âgée a déclaré:

Je vivais ma vie comme une routine. Je voulais faire semblant que rien n'était arrivé. Je passais tout mon temps avec mes amis. Papa me demandait pourquoi je ne restais pas à la maison, aux côtés de ma mère. Il ne me laissait pas aller à beaucoup d'endroits.

Une fille trouvait énervant que les gens lui demandent des nouvelles de sa mère parce que "ça lui faisait penser tout le temps à la situation" et une autre concentrait son attention sur son travail scolaire afin d'y échapper. Mais, bien qu'ils essaient de ne pas penser à la maladie, les enfants étaient nombreux à y revenir sans cesse. Une adolescente plus âgée a déclaré qu'elle essayait constamment de chasser le cancer de ses pensées mais que:

Cela ne fonctionnait pas vraiment parce que la maladie était toujours présente et qu'il y avait tant de choses à faire à la maison qu'on en était toujours angoissés et qu'on n'avait jamais plus envie de faire quoi que ce soit parce qu'on se sentait toujours dépassés.

Les enfants se tracassaient, avaient "les nerfs à vif" et certains finissaient par exploser. "Chaque petit détail m'inquiétait réellement et j'explosais parce qu'il ne fallait vraiment pas grand chose pour déclencher la tempête." Une jeune fille a également dit qu'elle était d'humeur exécrable:

Je me défoule toujours sur les autres. Je me souviens d'une fois où mon père m'a crié après sans raison évidente; je suppose que j'aurais dî comprendre qu'il avait beaucoup de soucis mais je ne l'ai pas fait. Je me suis mise en colère, moi aussi et je suis partie comme ça et ils ne savaient pas où j'étais. Mes crises de colère et mes fugues étaient fréquentes... Je devrais essayer de m'ouvrir plus et de prendre les choses en douceur afin d'épargner du stress à ma famille.

Qu'ils aient reconnu leur stress ou non, certains enfants utilisaient des mesures de réconfort telles que regarder la télévision, jouir d'une plus grande intimité physique et jouer avec leurs toutous favoris. Un garçon de 11 ans a déclaré: "ma vieille doudou, mon nounours et moi, on regardait la télé et alors je me sentais bien." Il semblait un peu gêné de se servir de tels jouets. Deux petites filles rapportaient que le bébé de la famille cessait de pleurer quand leur maman était à la maison et elles trouvaient du réconfort en s'asseyant sur les genoux de leur mère et en lui faisant des câlins. Quelques enfants se tournaient vers la prière ou la méditation. Certains enfants priaient seuls tandis que d'autres le faisaient en famille. Une fille a arrêté d'assister aux prières que sa famille tenait chaque soir. Le jeune adulte faisait appel à la prière et à la méditation parce qu'elles "l'empêchaient de se tracasser et lui permettait de garder l'esprit ouvert." Une petite fille croyait que ses prières étaient toujours exaucées:

Peut-être pas quand on s'y attend mais elles sont exaucées et je crois donc que cela aide... il y a quelqu'un en qui on peut avoir confiance et on espère que cette personne ne va pas nous laisser tomber, c'est bien l'unique personne en qui on peut avoir confiance et qui ne laisse jamais tomber.

Ainsi, les enfants reconnaissaient, à des degrés divers, le stress qui les habitait et le stress chez autrui. Alors que des familles reconnaissaient et exprimaient ouvertement le stress, d'autres pratiquaient surtout l'évitement. Les enfants se protégeaient ou étaient protégés de l'impact émotionnel de la situation.

\section{Partage}

Par "Partage", on entend la nature des conversations des enfants avec autrui sur la situation de leur mère et sur leurs inquiétudes ainsi que le degré avec lequel ils les tiennent. Certains faisaient preuve d'une grande ouverture alors que d'autres restaient plus renfermés. Ils se protégeaient émotivement en faisant attention à la manière dont ils parlaient à autrui, et les autres les protégeaient en veillant à ce qu'ils disaient et à la manière dont ils le disaient.

En général, les enfants ne parlaient ni de la situation ni de leurs inquiétudes avec quiconque, même pas avec leurs frères et soeurs, leurs amis et leurs camarades de classe. Le fait de ne pas en parler était pour eux une manière de se protéger émotionnellement. "Ce n'est pas facile pour moi d'exprimer mes sentiments tout haut. Dans ma tête, c'est différent." Une adolescente plus âgée parlait à ses amies les plus intimes dans une petite mesure parce qu'elles étaient aussi des "amies de la famille" mais c'est intentionnellement qu'elle taisait ses sentiments à l'école. Ce ne fut que plus tard qu'elle s'est confiée à son petit ami:

Je n'étais pas de glace mais je ne montrais rien de ce que je ressentais véritablement. J'essayais d'agir comme si de rien n'était. Je ne voulais pas l'annoncer au monde entier et je suppose que je n'étais pas la même personne à l'école. Mes deux grandes amies le savaient mais elles sont le genre de personnes avec qui je pouvais partager mes sentiments... mais il y a certaines choses qu'on ne peut pas faire, même avec sa meilleure amie. Nous ne tenions jamais de discussions approfondies. À la fin, j'avais un petit ami et je lui en ai parlé.

Il leur était difficile de parler de la maladie de leur mère et de leurs sentiments, et ils ne croyaient pas toujours que leurs amis les comprendraient. Ils taisaient leurs inquiétudes afin de ne pas en accabler les autres. Ils faisaient souvent de grands efforts pour cacher leurs sentiments et certains d'entre eux gardaient une distance physique et affective vis-à-vis de leurs amis. "C'est parfois très pénible et je ne voulais pas en accabler mes amis et les forcer à écouter tous mes problèmes." D'autres ont déclaré:

Je gardais tout cela en moi. Quand j'étais bouleversée, je pleurais ou bien je tapais dans un oreiller... Je ne l'ai dit à aucune de mes amies jusqu'à ce que la situation soit presque réglée et je n'avais donc personne avec qui parler, mais c'était de ma faute parce que j'étais mal à l'aise avec tout le monde.

Je me suis repliée sur moi-même. La seule personne avec qui je communiquais véritablement était ma meilleure amie à l'école. Je crois qu'il n'y a qu'elle. Nous étions d'excellentes amies en sixième année, un peu moins en septième et maintenant que nous sommes en huitième, elle est la seule à savoir absolument tout.

Une autre adolescente a dit qu'elle n'en parlait pas à sa meilleure amie et que cette dernière ne venait plus chez elle comme auparavant. Elle disait de la maison de son amie que c'était une "infirmerie" et elle ajoutait que quand on entrait dans la maison, on savait tout de suite que la maladie s'y était installée.

Les filles d'âge scolaire ont également affirmé qu'elles choisissaient les gens avec qui elles parlaient. "Nous parlions parfois avec nos amis quand nous étions chez eux et parfois aussi avec les adultes," mais c'était habituellement ces derniers qui lançaient la discussion en posant des questions. Les enfants n'abordaient pas le sujet d'eux-mêmes. L'un des garçons qui n'aimaient pas en parler avec ses amis ne pensait pas que son père ou que les autres membres de la famille en parlaient non plus. "Je ne pense pas que cela me plaise d'en parler maintenant (dans le cadre de cette étude). Cela ne me dérange pas en ce moment parce que j'aide." Même si les enfants ne le disaient pas à leurs camarades de classe, certains amis et camarades de classe pouvaient l'avoir appris par le biais de leurs propres parents. Un garçon a trouvé qu'un de ses camarades d'école était "plutôt curieux" lorsqu'il s'est enquis de la santé de sa mère. Il lui a donné une réponse des plus vagues. Il est possible que des parents aient demandé à leurs enfants de ne pas poser de questions 
sur la situation afin de protéger les sentiments des enfants touchés par le cancer de leur mère.

Par contre, la plus jeune des filles de la mère qui était en phase palliative a dit: "tout le monde le sait à l'école." On "en parle... on a une période pour la prière" et le fait d'en parler lui était d'un grand secours. Elle en avait également parlé avec ses deux meilleures amies. Lorsque les soeurs vont voir leur conseillère, "nous colorions et nous parlons de la situation et elle sort des livres sur les sentiments." Un autre enfant voyait les bénéfices du partage avec autrui sous un angle plus matérialiste. Plus il parlait avec de gens et "plus il recevait de cadeaux". Quelques enfants s'étaient vu demander s'ils voulaient parler à une conseillère et être dans un groupe incluant d'autres enfants dont la mère était également atteinte du cancer. Les jeunes adolescents étaient un peu plus réceptifs à l'idée alors que les adolescents plus âgés n'y étaient pas du tout. "Qui a envie d'en parler? Maman se sent terriblement mal aujourd'hui. Elle a l'air mal en point. La maison est un vrai désastre. Ma vie est merdique. Ce n'est pas une bonne raison de se réunir avec d'autres jeunes." Bien que cette adolescente jure qu'elle ne voulait pas parler à une conseillère, elle a déclaré qu'il n'était pas impossible qu'elle parle à quelqu'un d'autre. Elle avait parlé à une conseillère et avait trouvé cela inutile."

Ça ne m'a pas beaucoup aidée parce je ne suis pas du genre à parler à une personne que je ne connais pas...c'était instructif mais à part ça, ça ne m'a rien apporté. Je suis allée une fois (au centre de cancer) mais les médecins ne vous parlent pas vraiment. (Elle ne voulait pas parler à un membre de la famille parce que) quelque chose pourrait lui échapper et elle serait furieuse si la personne était incapable de le garder secret, mais à quelqu'un avec qui on est à l'aise pour parler... et on pourrait lui dire ce qui nous tracasse, de préférence une personne qui a déjà vécu cette situation serait beaucoup mieux parce qu'elle pourrait se mettre à notre place. (Elle ne souhaitait pas parler à un conseiller parce que) c'est comme si on s'adressait à un parfait inconnu - on ne s'ouvre pas normalement parce qu'on ne connaît pas la personne et qu'on ne va pas lui confier son secret le plus cher ou ses inquiétudes.

Le jeune adulte a déclaré que le fait de parler avec des pairs serait utile si cela se faisait dans le cadre d'un groupe situé non loin de chez lui. Le fait de parler au téléphone à une personne de son âge qui aurait vécu une situation semblable pourrait lui être utile et cela serait plus facile puisqu'il n'aurait pas besoin de quitter sa demeure. Les pairs qui pouvaient lui apporter du soutien se trouvaient dans une autre ville mais il ne semblait pas qu'il ait parlé de la situation de sa mère avec eux.

Les enfants ont indiqué si on abordait le sujet du cancer à la maison et comment leur famille parlait du cancer avec autrui. Certaines familles voulaient que cela reste dans la famille alors que d'autres en parlaient autour d'elles. Dans une famille particulière, il était acceptable d'aborder le sujet avec des membres de leur paroisse tandis qu'elle en décourageait la discussion avec les gens de l'extérieur. Dans une autre famille, on encourageait la jeune adolescente à en parler. “Maman m'a dit que si j'éprouvais des sentiments que je ne pouvais pas aborder avec elle, je pourrais en parler à mon ancienne enseignante parce qu'elle était une des meilleures enseignantes que j'ai jamais eues... J'étais plus à l'aise avec elle qu'avec ma mère pour parler de ce sujet. C'était plus facile et maintenant nous nous sommes liées d'amitié et avons abandonné la relation enseignante-élève." Cette jeune fille n'en a pas parlé à ses amies. Une adolescente a déclaré ce qui suit à propos de la communication familiale:

Je crois que la plupart des membres de la famille le gardaient en eux. Ils n'exprimaient ni leurs sentiments véritables ni leurs peurs. Ils intériorisaient leurs émotions et je crois que nous aurions dî en parler davantage parce que maintenant, si on examine les choses, il est vraiment difficile d'en parler. Si quelque chose nous tracasse, on n'a pas l'habitude d'en parler avec d'autres personnes - c'est notre problème après tout règle-le toi-même, plutôt que d'être ouvert aux autres, de leur parler et de leur dire ce qui nous chiffonne.
Quoique le cancer du sein soit une maladie grave, les enfants y ont trouvé des notes humoristiques qui présentent autant de possibilités de partage. Deux petites filles "jouaient avec le faux sein de maman - comme si c'était un sac de fèves," et bien que cela n'enchante guère leur maman, cette dernière ne le leur interdisait pas.

Quelques parents préparaient le terrain en s'entretenant avec les enseignants de leurs enfants et en leur révélant ce qui se passait. "Mes enseignants ont toujours été au courant. Ils ne cessaient de me demander de ses nouvelles." Ce garçon pensait aussi que sa mère avait rédigé un mot à son enseignant et que son père avait averti son entraîneur de hockey. Il semble que les enfants trouvaient utile que d'autres personnes sachent ce qui se passait et qu'elles utilisent l'information de façon salutaire.

La majorité des enfants ne parlaient donc pas de la situation à autrui afin de se protéger émotivement et de protéger les autres. Ils ne croyaient pas que leurs amis comprendraient et ils avaient du mal à en parler. Dans de nombreuses familles, le partage restait minime.

\section{Changement/Aide}

Le thème "Changement/Aide" reflète dans quelle mesure les comportements habituels évoluaient et de quelle manière les enfants apportaient leur aide. L'âge des enfants, la disponibilité d'un soutien familial et de ressources, les comportements habituels antérieurs de la famille et sa composition ainsi que la gravité de la maladie de la mère influaient tous sur l'évolution des routines de vie et sur le nombre de tâches ménagères effectuées par les enfants. Les enfants étaient protégés de l'impact émotionnel si les routines ne changeaient pas beaucoup. Certains parents prenaient des mesures importantes dans le but de minimiser les changements sur le plan des habitudes de vie, particulièrement pour les enfants.

Quelques enfants n'ont pour ainsi dire pas remarqué de changements au niveau de leurs routines de vie. Pour d'autres, l'impact de la situation était majeur. Un petit garçon "jouait tout simplement" comme il le faisait habituellement et d'autres enfants qui affirmaient ne pas être très conscients de ce qui se passait poursuivaient leurs activités comme par le passé. L'impact qu'ils ressentaient était restreint et ils ne s'inquiétaient pas outre mesure. Un adolescent a déclaré que "la vie continuait." Lorsqu'il demandait à sa mère comment elle allait après avoir suivi un traitement de chimiothérapie, elle lui répondait habituellement 'bien' et "les choses n'allaient guère plus loin." Il réalisait que ses parents prenaient la situation bien plus sérieusement que lui et il a ajouté qu'il leur offrirait, dorénavant, davantage de soutien.

Quoique certains enfants soient plutôt flexibles et reconnaissent l'accroissement des exigences et la nécessité de l'évolution des routines de vie, le changement n'en ait pas moins stressant. Deux soeurs d'âge scolaire ont dit: "Nous ne pouvons plus faire grandchose avec maman.. elle doit se reposer pendant longtemps avant d'assister à nos concerts et à nos présentations aux foires scientifiques et tout et tout." Leurs grands-parents sont venus à la rescousse et elles allaient donc chez eux mais elles ne voulaient pas y aller, en dépit de l'amour qu'elles ressentaient à leur égard. Pour d'autres enfants, le fait que le père passe davantage de temps au foyer n'était pas toujours une sinécure. Il "ne parvenait pas à empêcher le bébé de pleurer et il nous obligeait à aller au lit de bonne heure." Il leur interdisait de regarder la télévision comme ils en avaient l'habitude, en partie parce qu'il désirait y regarder des épreuves sportives.

Les enfants remarquaient l'évolution des routines de vie et même les ajustements minimes étaient pénibles et bouleversants. Cela les gênait que leur mère ne soit pas là pour leur souhaiter une bonne nuit ou que les collations ne soient pas prêtes comme d'habitude. Un enfant a dit que "c'était bizarre" lorsque c'était des gens autres que sa mère qui conduisaient parce qu'elle avait l'habitude de s'arrêter dans divers magasins sur le chemin du retour. Dans une famille, les adolescents trouvaient pénible de devoir demander de l'argent à leur père parce que "il n'est jamais au courant à propos de l'argent - au point de nous demander pourquoi on a besoin d'argent!" 
D'énormes efforts étaient fournis pour que les routines de vie restent aussi normales que possible. D'autres membres de la famille et des amis prêtaient main forte. "Ma grand-mère a plus ou moins pris sa place et ça a résolu le problème. (Grâce à son aide), je dirais que les choses n'ont pas changé." Quelques familles ont embauché quelqu'un, ce qui a allégé le fardeau en partie. "Avant, ma maman faisait tout le travail à la maison et quand elle était malade, la femme de ménage faisait tout à sa place et papa n'avait donc rien à faire." Par contre, le fait d'embaucher quelqu'un pour aider n'était pas nécessairement sans problèmes:

(la femme de ménage) était surprotectrice... ce n'était plus notre maison à nous. Elle essayait de rendre service mais cela m'embêtait... l'idée qu'il fallait qu'elle soit là. En sa présence, on ne peut pas vraiment se trimbaler en petite culotte sans se sentir gênés... enfin, on s'habitue à elle. Elle faisait manger maman quand elle n'en avait pas envie.

Une adolescente déclarait qu'elle avait vécu son adaptation au changement de manière positive mais qu'elle s'inquiétait du fait que les choses ne seraient plus jamais comme avant. Elle jugeait qu'elle avait réussi à "faire face aux changements et à les accepter et à s'adapter à ses nouvelles habitudes de vie et à reconnaître que sa mère n'est plus comme avant et qu'elle ne le sera jamais plus."

Aucune aide spéciale n'était attendue de certains enfants tandis que d'autres assumaient des responsabilités importantes et se chargeaient de tâches majeures. Dans certains cas, ces dernières s'avéraient pénibles à cause des compétences et du temps qu'elles exigeaient, ce qui empêchait les jeunes de faire des choses "normales" et d'être avec leurs pairs. Ils ne voulaient pas toujours être chez eux et préféraient être avec des amis, mais ils étaient nombreux à penser qu'ils devraient être auprès de leur mère à se sentir coupables lorsqu'ils n'y étaient pas. "Je ne sortais pas tant que ça de toute façon parce que je voulais rester aux côtés de maman et puis, la seule fois où je sors, cela se termine mal. Je crois que je ne devrais plus sortir. Je me sentais coupable."

Dans certaines familles, l'aide des enfants était essentielle. Plutôt que maintenir la normalité des routines de vie en comptant sur l'intervention de gens de l'extérieur, on demandait aux enfants, qui n'avaient parfois pas plus de sept ans, d'aider à maintenir les activités quotidiennes. Ainsi, les enfants devaient abandonner certaines activités dans d'autres domaines et participer à des tâches liées au soin de l'enfant ou au ménage ou même en assumer l'entière responsabilité. Plusieurs enfants trouvaient que leurs nouvelles responsabilités étaient pénibles. Deux petites filles d'âge scolaire ont déclaré: "Parfois, c'était dur parce que nous devions nous occuper de notre petite soeur... Quelquefois, nous n'arrivions pas à l'empêcher de pleurer continuellement et papa avait l'air fâché après nous." La cadette devait faire du ménage et mettre le couvert, réchauffer le lait de sa petite soeur et préparer ses propres collations. Si elles refusaient, leur père se mettait "vraiment en colère... On ne pouvait presque pas jouer avec nos amis parce que papa disait toujours non." Des adolescents ont décrit leurs nouvelles responsabilités. "Les plus jeunes ne pouvaient pas en faire autant que moi, alors comme je suis l'aînée, il faut savoir assumer ses responsabilités." Une jeune adolescente qui se chargeait des travaux que sa mère accomplissait auparavant avait dû délaisser des activités qu'elle adorait et son travail scolaire "en avait pris un sacré coup:"

J'ai grandi en un rien de temps parce que tout d'un coup, on attend de toi que tu fasses bien des choses et que tu n'en as pas l'habitude, mais tu t'adaptes... Tu ne te conduis plus comme un enfant de 12 ans ordinaire. Tes pensées et tout le reste diffèrent totalement de ceux des enfants de ton âge parce que tu as déjà vécu tant de choses... C'est comme si tout le reste n'était plus si important que ça.

Un garçon plus âgé n'était guère à l'aise avec le rôle d'assistant qu'il devait jouer. Il participait aux tâches ménagères, aidait à faire la cuisine, à faire les courses et à conduire sa mère à ses traitements. "Il faut que je passe le plus clair de mon temps à la maison et ça, ce n'est pas dans mes habitudes." Il préférait que les gens qui leur rendaient visite restent ailleurs.
Il était difficile pour certains enfants de composer avec toute l'attention dont la mère faisait l'objet. "Certes, on est supposés se préoccuper de la santé de cette personne, mais cela m'embêtait parce que personne ne me demandait comment j'allais, moi, alors que les gens demandent tous des nouvelles de la malade." Cela ennuyait quelques enfants que les gens demandent sans cesse des nouvelles de leur mère: "Ne me posez plus la question."

Les enfants subissaient donc un impact majeur lorsque les routines de vie évoluaient. Quoique beaucoup de familles aient tenté de minimiser la fluctuation des routines de vie et de protéger émotionnellement les enfants et elles-mêmes, même les changements les plus subtils revêtaient une grande signification pour les enfants. Pour que la vie quotidienne se poursuive, on demandait souvent aux enfants d'aider et d'assumer des tâches et des responsabilités importantes. Ils n'avaient aucune protection émotionnelle contre l'impact de la situation.

\section{Discussion et répercussions}

Pour la majorité des enfants, le cancer et la chimiothérapie de la mère exercent un impact considérable qui provient notamment de ce qu'ils voient, entendent et lisent, de la coloration affective perçue dans la famille et de l'évolution des comportements. La protection émotionnelle de soi et des autres prend plusieurs formes. Dans une mesure plus ou moins grande, l'attitude protectrice émanant des enfants et autres personnes, principalement le parent, joue un rôle majeur sur le plan de l'adaptation des enfants. Étant donnée la nature stressante du cancer et de la chimiothérapie et des répercussions des circonstances, le phénomène d'autoprotection émotionnelle se produit fréquemment afin de minimiser l'impact de la situation. Il n'y a pas que les enfants qui se protègent émotionnellement eux-mêmes en se mettant en quête d'information ou en évitant toute information et en partageant ou non leurs inquiétudes, mais les autres protègent émotivement les enfants en leur disant ou non ce qui ce passe, en choisissant ce qu'ils leur apprennent et en s'efforçant ou non de maintenir le fonctionnement familial normal.

Des schémas de protection émotionnelle semblables à ceux que nous avons soulignés dans la présente étude ont également été signalés dans l'étude de Brown (1992) concernant des adolescents aux prises avec le cancer avancé d'un de leurs parents. Ils faisaient appel à l'autoprotection émotionnelle pour atténuer la peur qu'ils ressentaient face à l'avenir et pour pouvoir s'atteler aux tâches qui consistent à se séparer de la famille et à emprunter la voie de l'autonomie et de l'indépendance. Ils se protégeaient émotivement en s'efforçant de ne pas penser à la situation au foyer, en passant du temps avec leurs amis pour se couper de ce qui se passait chez eux, en essayant de garder en tête l'ancienne image qu'ils avaient du parent atteint, en espérant que le parent allait se rétablir et en gardant une attitude positive vis-à-vis de sa guérison éventuelle. Bien qu'on ne leur ait pas donné les mêmes noms, l'autoprotection et la protection émotionnelles ont également été dégagées dans d'autres études. Hilton et Elfert (1996) décrivaient comment les parents adaptaient ce qu'ils disaient aux enfants afin de minimiser l'impact de la situation et les efforts majeurs qu'ils fournissaient pour garder les routines de vie des enfants aussi normales que possible afin d'atténuer le stress. McTaggart (2000) décrivait la sollicitude et l'attitude protectrice mutuelles de la mère et de sa fille qui avaient pour but de minimiser la menace envers elle-même et envers l'autre. Des stratégies communément employées étaient le maintien d'un sentiment de normalité et la limitation du nombre de conversations sur l'expérience entourant le cancer, notamment en ce qui a trait au pronostic et à l'éventualité de la mort. Le thème "La vie continue" de l'étude d'Issel et al. (1990) montrait comment les enfants minimisaient l'importance de la maladie en faisant comme si de rien n'était et en continuant de faire ce qu'ils avaient l'habitude de faire.

On constatait d'importances variations sur le plan de l'autoprotection et de la protection émotionnelles qui tenaient aux éléments suivants: âge et niveau de développement, gravité relative de la maladie de la mère, impact de la maladie sur les activités de cette 
dernière, composition de la famille, routines de vie antérieures, disponibilité de personnes prêtes à aider et ressources financières de la famille. Quelques enfants ont bénéficié d'une protection considérable afin qu'ils ne prennent pas vraiment conscience de la gravité de la situation par le biais de ce que l'on révélait et de ce qu'ils observaient relativement à l'évolution des routines de vie. Le fait d'être protégé émotionnellement influait de manière prépondérante sur le comportement des enfants. Si les enfants ne percevaient aucun signe de stress ou ne remarquaient pas de changements évidents chez la mère et à la maison, ils ne ressentaient pas la nécessité de se protéger émotivement. La plupart des enfants avaient été mis au courant de la situation et peu d'entre eux avaient été exposés à une dose massive d'information. Il était inhabituel que les enfants recherchent activement des renseignements en vue de compléter ce qu'on leur avait dit à moins qu'ils n'acquièrent de l'information de manière passive en provenance d'autrui, du matériel scolaire, d'émissions de télévision et d'Internet.

La protection émotionnelle diffère de la négation de la réalité qui, d'après Kalish (1985), est un comportement d'évitement inconscient visant à protéger le moi de l'impact émotionnel lié à l'éventualité bien réelle de la mort. La protection émotionnelle est un moyen d'atténuer la peur concernant l'avenir qui permet aux enfants de poursuivre leur cheminement dans la vie. En elles-mêmes, l'autoprotection et la protection émotionnelles ne sont ni positives ni négatives, notamment lorsqu'elles font l'objet d'une utilisation modérée. Par contre, un emploi excessif ou insuffisant pourra entraîner des problèmes. Ceuxci peuvent survenir lorsque les enfants se sentent mal informés ou qu'ils ne sont pas dans le bain à cause d'une protection émotionnelle excessive ou lorsqu'ils se sentent dépassés par les événements et peu préparés à y faire face du fait d'une protection émotionnelle insuffisante ou inadéquate.

Elsegood (1996) décrit les vues divergentes entourant la question, à savoir s'il convient ou non d'annoncer la mauvaise nouvelle aux enfants: 'l'approche protectrice' qui vise à protéger émotivement les enfants en leur taisant la mauvaise nouvelle et 'l'approche franche' selon laquelle il faut que les enfants sachent. La prise de décision se complique davantage quand la mauvaise nouvelle est associée à des sentiments de honte ou qu'elle s'accompagne d'incertitudes. Elsegood souligne qu'il n'est pas nécessaire que la divulgation soit un cas de 'tout ou rien' et que la franchise et l'ouverture totale ne sont pas synonymes. De plus, il importe de reconnaître que les enfants deviennent conscients des mauvaises nouvelles ou du fait qu'il y a quelque chose qui "cloche" même lorsqu' on ne les a pas mis ouvertement au courant de la situation ou qu'on leur cache l'information. Quoique les enfants ressentent de la détresse lorsqu'ils apprennent des mauvaises nouvelles, il est probable qu'ils en ressentent moins lorsque l'information leur est communiquée de façon claire et franche (Jewett, 1994). L'argument selon lequel les enfants n'ont pas besoin qu'on leur dise ce genre de choses parce qu'ils n'ont pas la capacité de comprendre des concepts abstraits sous-estime l'influence qu'exerce le vécu de l'enfant sur son niveau de compréhension. Whittham (1993) affirme que les enfants peuvent s'impliquer activement et peuvent tirer des bienfaits de l'aide qu'ils se donnent lorsqu'on les met au courant de la situation en toute franchise. La divulgation est perçue comme étant bénéfique (Rosenheim et Reicher, 1985). Elsegood souligne qu'une fois qu'on a annoncé la mauvaise nouvelle aux enfants, ceux-ci peuvent et devraient avoir l'occasion de prendre une part active à la décision concernant ce qu'ils aimeraient apprendre d'autre. Il ajoute que les gens qui suggèrent qu'il ne faut dire les choses aux enfants que lorsqu'ils demandent à savoir ou semblent vouloir savoir comptent trop sur la capacité des enfants à s'exprimer et sur la capacité des gens à reconnaître les expressions voilées. Il avance que ces enfants-là se trouveraient alors dans un état d'isolement, de confusion et d'anxiété jusqu'à ce qu'ils soient capables de s'exprimer ou que l'on reconnaisse leurs peurs et inquiétudes cachées. Elsegood décrit également comment les enfants essaient souvent de protéger leurs parents pour empêcher qu'ils ne soient bouleversés et essaient aussi de se protéger de la réaction qu'ils anticipent chez leurs parents.
La protection émotionnelle était largement sous l'influence de la nature et du degré de changement que les routines des enfants devaient subir. Le fait de devoir changer les routines de vie est extrêmement stressant car les enfants y tenaient fermement. D'autres auteurs ont fait remarquer qu'il importait de maintenir la normalité de la vie (Hilton, 1996; Issel et al., 1990). Si certaines familles réussissaient à minimiser ou à réduire les perturbations, d'autres vivaient des changements majeurs. Lorsque la mère était incapable, du fait de la chimiothérapie, d'assumer normalement ses rôles habituels, particulièrement à la maison, la situation avait un impact considérable sur la famille et une incidence significative sur l'enfant. C'est ce qui arrivait aux enfants qui devaient assumer des responsabilités importantes au foyer afin d'entretenir la vie familiale. Ils ne se sentaient pas protégés, émotivement et autrement, et sentaient qu'ils avaient atteint leurs limites, et souvent, qu'ils étaient mal préparés pour les responsabilités qu'ils avaient dû assumer. Un plus grand nombre de filles que de garçons a assumé des responsabilités relatives aux tâches ménagères ou au soin de l'enfant, mais ce phénomène ne s'avérait pas toujours vrai. L'école procurait aux enfants un contexte normalisateur ou même peut-être un refuge. Quand les mères suivaient leur chimiothérapie durant l'été lorsque les enfants étaient en vacances, c'était 24 heures sur 24 que ces derniers étaient exposés à l'impact du cancer. Bien que la souplesse de leur emploi du temps estival constitue un avantage, ils ne pouvaient pas compter sur les schémas réguliers de l'école pour échapper à la lourdeur de la situation.

S'ils voulaient maintenir la normalité de la vie, les enfants pouvaient essayer, entre autres, de chasser la situation de leurs pensées et de ne pas y porter attention. Les enfants évitaient intentionnellement d'en parler avec les autres. Il était fréquent que les enfants se protègent émotivement en ne parlant pas de leurs peurs aux autres ou en faisant extrêmement attention à ce qu'ils disaient. Certes ils ne savaient pas quoi dire, mais ils ne pensaient pas que leurs amis comprendraient et donc ils ne parlaient pas avec eux de leurs inquiétudes. Ils ne voulaient pas causer de l'anxiété à autrui en abordant le sujet. Garder le silence était un peu comme le prolongement de certaines habitudes familiales en vertu desquels on ne discute pas de la situation. Et quoiqu'ils ne partagent ni leurs sentiments ni leurs préoccupations, le stress se lisait dans leur comportement et dans celui de leur famille. Certains enfants avaient conscience de leurs réactions émotionnelles et comportementales face à la situation ainsi que de celles d'autres personnes, notamment au sein de la famille, et ils pouvaient ou non avoir modifié leur propre comportement en conséquence. Il se peut que d'autres enfants aient reconnu les niveaux accrus de stress chez eux-mêmes et chez autrui, mais cela ne les empêchait pas de continuer à réagir face à la situation et aux autres. On retrouve ces mêmes éléments dans d'autres études, notamment dans celle de McTaggart (2000) dont un des thèmes était Intention et comédie.

Il est impossible de cerner l'impact à long terme, mais certains des enfants de l'étude subissaient l'impact de la situation créée par la maladie longtemps après la fin du traitement, particulièrement ceux qui avaient été exposés aux dures réalités et intensités qui s'y rapportaient. Il n'était pas rare que certains éprouvent initialement de la rancour tandis que d'autres acquéraient de nouvelles perspectives sur eux-mêmes par le biais de la maladie de leur mère. La nécessité de grandir rapidement a été signalée, particulièrement par les enfants qui avaient dû assumer de lourdes tâches ménagères.

Il convient d'examiner les conclusions de l'étude à la lumière des limites de la recherche. L'échantillon était de petite taille et d'une diversité culturelle minime. La réalisation d'entrevues auprès d'un plus grand nombre d'enfants aurait pu fournir des renseignements additionnels et étoffer les thèmes. Les conclusions suggèrent que la protection émotive chez les enfants et les familles revêt une grande importance et qu'une utilisation inadéquate, excessive ou insuffisante de l'autoprotection et de la protection émotionnelles peut s'accompagner de problèmes. Les conclusions renferment également des répercussions pour la pratique clinique et la recherche. Il nous 
incombe, en tant que professionnelles de la santé, de faire une différence dans la vie des enfants et des familles. Plusieurs recommandations concernant les enfants, les parents et les prestataires de soins sont présentées, illustrant les manières dont il convient d'informer les enfants et de les impliquer dans les situations où la mère suit une chimiothérapie (voir le tableau deux).

\section{Remerciements}

Les auteures tiennent à exprimer leur appréciation de l'aide apportée par les autres co-investigatrices à la planification, à la mise en oeuvre et à l'analyse de la grande étude (J. Brown, A. Ho, G. Heaps, E. Holwerda, M. Rechner, S. Sample et G. Tennant), par les autres assistants de recherche (L. Esson, P. Fisher, A. Lee et L. Unger) et par les femmes, les hommes et les enfants qui ont participé à l'étude. Cette dernière a reçu l'appui financier de la Fondation canadienne pour le cancer du sein.

Tableau deux: Recommandations concernant les enfants, les parents et les professionnels

de la santé et visant à favoriser l'adaptation des enfants dont un parent a le cancer

Enfants

Sensibiliser les enfants et les aider à avoir une conscience accrue de la situation et à mieux la comprendre - mettre à la disposition des parents et des enfants du matériel dans divers formats (vidéo, documentation écrite, ordinateur, Internet) en tenant compte de l'âge, du niveau de développement et de la culture

- encourager la participation à des programmes offerts par le centre de cancer/les centres de traitement; p. ex. thérapie par l'art, séances destinées aux enfants

Aider les enfants dans leur adaptation émotionnelle à la situation - aider les enfants à réaliser que des réactions telles que la colère, la peur et la culpabilité sont normales tout comme l'est l'utilisation de mesures de réconfort, p. ex. toutous et doudous

- encourager d'autres manières de réduire le stress

- aider les enfants à trouver le juste équilibre entre les demandes émanant de la famille et le fait de n'être encore "que des enfants." Il faudra peut-être négocier avec les parents afin de trouver un équilibre et d'atténuer le sentiment de culpabilité des enfants lorsqu'ils ne sont pas au foyer

- les encourager à parler de leurs inquiétudes et à parler avec d'autres enfants qui ont vécu une situation similaire.

- la mise sur pied de groupes d'entraide en oncologie peut s'avérer utile.

\section{Parents}

Aider les parents à savoir ce qu'il faut dire aux enfants et comment

- identifier le matériel-ressource qui convient

- aider les parents à composer avec leur propre situation au sein de groupes de soutien

- encourager une franchise raisonnable: signaler ce qui convient à différents groupes d'âge

- aider les parents à être à l'affût d'éventuels comportements régressifs chez leurs enfants

- encourager les parents à amener leurs enfants au centre de cancer

Aider les parents à comprendre les réactions courantes chez les enfants

- tenir des séances examinant les réactions les plus courantes des enfants de différents groupes d'âge

- sensibiliser les parents à l'aversion des enfants envers le changement et les aider à faire la distinction entre ce qui doit changer et ce qui peut demeurer plus stable

Aider les parents à travailler avec les réactions émotionnelles de l'enfant

- les encourager à fournir à leur famille des occasions propices au partage

- sensibiliser les parents à l'existence des ressources destinées aux enfants, p. ex. musicothérapie, thérapie par l'art, conseillères

- encourager les parents à discuter des pratiques qui peuvent être soit troublantes soit pleines de sens pour les enfants et chercher à les résoudre, le cas échéant; p. ex., lorsque la mère est hospitalisée, garder sa photo à proximité et lui demander de téléphoner tous les soirs à la maison pour souhaiter bonne nuit aux enfants

Aider les parents à savoir comment ils peuvent impliquer les enfants et ce qu'il est raisonnable d'attendre d'eux en matière de partage des tâches à la maison

- les aider à réaliser ce qui peut convenir aux enfants ou au contraire être trop accablant pour eux en examinant la situation, les pratiques passées, les croyances et routines de vie familiales

- aider les enfants à comprendre leur propre valeur et l'importance de la réalisation des tâches qui leur sont confiées

- encourager les parents à prévenir les enseignants de la situation afin qu'ils soient mieux préparés.

Infirmières et autres professionnels de la santé:

Favoriser la préparation des professionnels de la santé dans leur travail auprès des parents et des enfants

- organiser des programmes de perfectionnement sur place axés sur les besoins de la famille et des enfants et sur les façons dont on peut travailler avec eux

- accroître leur sensibilisation au matériel et aux ressources - et à leur qualité - qu'ils peuvent recommander aux parents et aux enfants auprès desquels ils oeuvrent

- développer des programmes/systèmes qui peuvent aider les parents et les enfants dans leur cheminement tels que la création d'un système de "pairage" où les femmes peuvent bénéficier de l'appui d'autres femmes et où les conjoints/partenaires et les enfants peuvent parler avec des pairs

- évaluer les besoins d'information des parents et leur besoin de soutien relativement à leurs enfants

- faciliter l'accès à l'information en dégageant pour eux diverses manières dont ils peuvent trouver des informations utiles

- incorporer la participation des enfants lorsque c'est pertinent et faisable

- aider, dans la mesure du possible, à réduire les perturbations et à favoriser la continuité en sensibilisant les parents aux ressources qui pourraient les y aider comme les services disponibles de soin à l'enfant au centre de cancer ou dans le quartier

- des études additionnelles doivent se pencher sur les réactions des enfants à la maladie d'un de leurs parents et ce depuis la perspective des enfants, et d'autres mettre à l'essai les interventions mentionnées ci-dessus 
Références

Birenbaum, L.K., Yancey, D.Z., Phillips, D.S., Chand, N., \& Huster, G. (1999). School-age children's and adolescents' adjustment when a parent has cancer. Oncology Nursing Forum, 26(10), 1639-1645.

Brown, J. (1992). The experience of teenagers living with a parent with advanced cancer. Unpublished Master's thesis, University of British Columbia, Vancouver, BC.

Compas, B.E., Worsham, N.L., Epping-Jordan, J.E., Grant, K.E., Mireault, G., Howell, D.C., \& Malcarne, V.L. (1994). When mom or dad has cancer: Markers of psychological distress in cancer patients, spouses, and children. Health Psychology, 13, 507-515.

Denzin, N., \& Lincoln, Y. (Eds.). (1994). Handbook of qualitative research. Thousand Oaks, CA: Sage.

Elsegood, J. (1996). Breaking bad news to children. In B. Lindsay \& J. Elsegood (Eds.), Working with children in grief and loss (pp. 32-55). London, UK: Bailliere Tindall.

Erlandson, D.A., Harris, E.L., Skipper, B.L., \& Allen, S.D. (1993). Doing naturalistic inquiry. Newbury Park, CA: Sage.

Hilton, B.A. (1996). Getting back to normal: The family experience during early stage breast cancer. Oncology Nursing Forum, 23(4), 605-614.

Hilton, B.A., Brown, J., Ho, A., Holwerda, E., Rechner, M., \& Sample. S. (1996). Creative intervention report: Developing creative interventions to assist young families to meet the challenges of chemotherapy for breast cancer. Unpublished manuscript, University of British Columbia at Vancouver.

Hilton, B.A., \& Elfert, H. (1996). Children's experiences with mothers' early breast cancer. Cancer Practice, 4(2), 96-104.

Howe, M.J., Hoke, I., Winterbottom, M., \& Delafield, D. (1994). Psychosocial effects of breast cancer on patient's children. Journal of Psychosocial Oncology, 12, 1-21.

Hymovich, D.P. (1993). Child-rearing concerns of parents with cancer. Oncology Nursing Forum, 20, 1355-1360.

Issel, L.M., Ersek, M., \& Lewis, F.M. (1990). How children cope with mother's breast cancer. Oncology Nursing Forum, 1(suppl.), 5-13.

Jewett, C. (1994). Helping children cope with separation and loss. London, UK: Batsford.

Kalish, R.A. (1985). The final transition. Farmingdale, NY: Baywood, Publishing Co.
Lewis, F.M., Zahlis, E.H., Shands, M.E., Sinsheimer, J.A., \& Hammond, M.A. (1996). The functioning of single women with breast cancer and their school-aged children. Cancer Practice, 4, 15-24.

Lichtman, R.R., Taylor, S.E., Wood, J.V., Bluming, A.Z., Dosik, G.M., \& Leibowitz, R.L. (1984). Relations with children after breast cancer. The mother-daughter relationship at risk. Journal of Psychosocial Oncology, 2(3/4), 1-19.

Lincoln, Y., \& Guba, E. (1985). Naturalistic inquiry. Beverly Hills, CA: Sage.

McTaggart, D.L. (2000). Breast cancer experience: Mothers, adolescent daughters and the mother-daughter relationship. Doctoral dissertation, University of British Columbia, Vancouver.

Nelson, E., Sloper, P., Charlton, A., \& While, D. (1994). Children who have a parent with cancer: A pilot study. Journal of Cancer Education, 9(1), 30-36.

Rosenfeld, A., \& Caplan, G. (1983). Adaptation of children of parents suffering from cancer: A preliminary study of a new field for primary prevention research. Journal of Primary Prevention, 3, 244-250.

Rosenheim, E., \& Reicher, R. (1985). Informing children about a parent's terminal illness. Journal of Child Psychology and Psychiatry, 26, 995-998.

Shands, M.E., Lewis, F.M., \& Zahlis, E.H. (2000). Mother and child interactions about the mother's breast cancer: An interview study. Oncology Nursing Forum, 27(1), 77-85.

Strauss, A., \& Corbin, J. (1990). Basics of qualitative research: Grounded theory procedures and techniques. Newbury Park, CA: Sage.

Wellisch, D.K., Gritz, E.R., Schain, W., Wang, H., \& Siau, J. (1991). Psychological functioning of daughters of breast cancer patients: Part I: Daughters and comparison subjects, Psychosomatics, 32(3), 324-336.

Wellisch, D.K., Gritz, E.R., Schain, W., Wang, H., \& Siau, J. (1992). Psychological functioning of daughters of breast cancer patients: Part II: Characterizing the distressed daughter of the breast cancer patient. Psychosomatics, 33(2), 171-179.

Whittham, E.H. (1993). Terminal care of the dying child. Psychosocial implications of care. Cancer, 71, 3450-3462.

Zahlis, E.H. (2001). The child's worries about the mother's breast cancer: Sources of distress in school-age children. Oncology Nursing Forum, 28(6),1019-1025. 